\title{
Molecular detection and quantification of Plasmodium falciparum gametocytes carriage in used RDTs in malaria elimination settings in northern Senegal
}

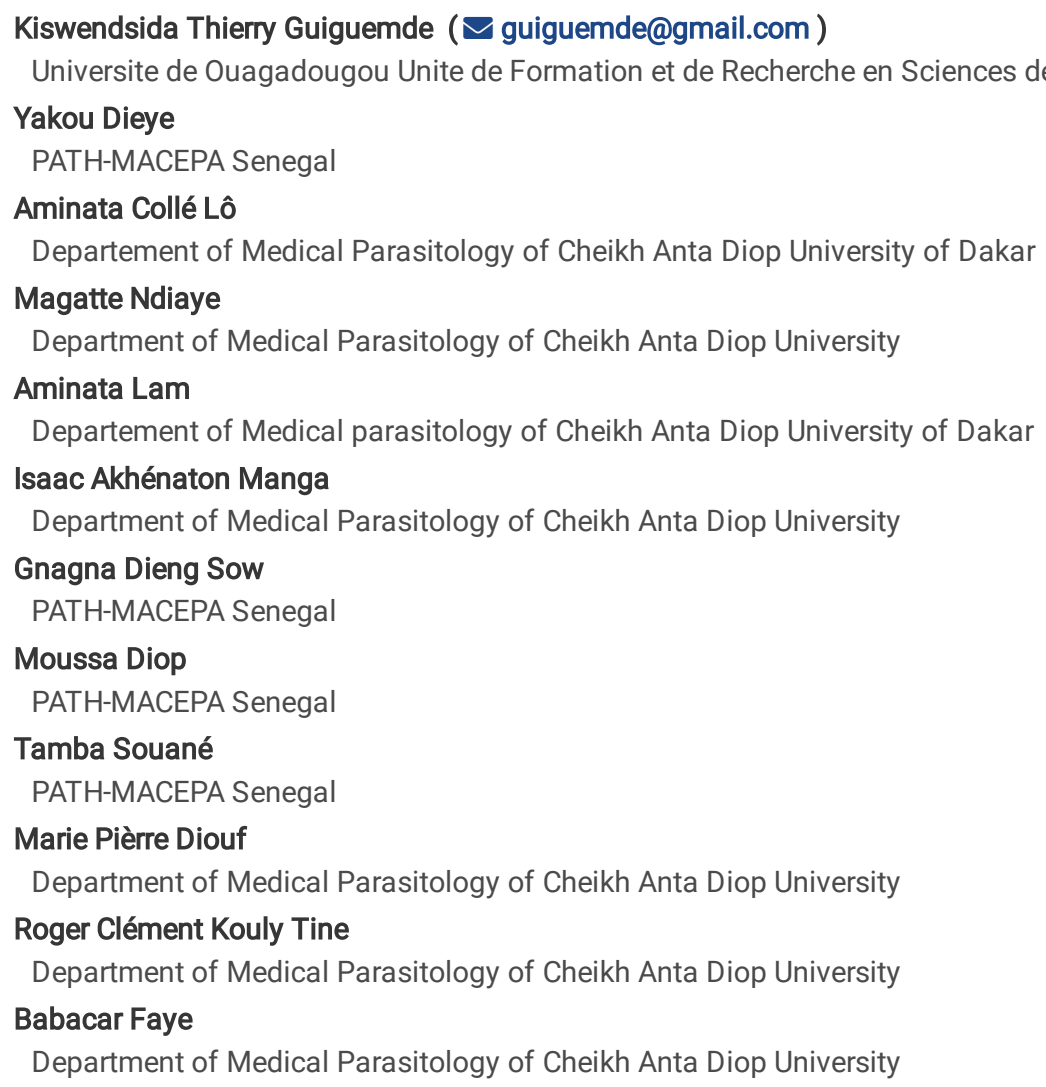

\section{Research}

Keywords: Malaria, RDT, Gametocytes, DNA extraction, Quantification, Plasmodium falciparum, qPCR.

Posted Date: February 18th, 2020

DOI: https://doi.org/10.21203/rs.2.17896/v2

License: (9) This work is licensed under a Creative Commons Attribution 4.0 International License. Read Full License

Version of Record: A version of this preprint was published at Malaria Journal on March 30th, 2020. See the published version at https://doi.org/10.1186/s12936-020-03204-w. 


\section{Abstract}

Background: Malaria surveillance requires powerful tools and strategies to achieve malaria elimination. Rapid diagnostic tests for malaria (RDTs) are easily deployed on a large scale and are helpful sources for the parasite's DNA. The application of sensitive molecular techniques to these RDTs is a modern tool for improving malaria case detection and drug resistance surveillance. Several studies have made it possible to extract the DNA of $P$. falciparum on these RDTs. The knowledge of gametocyte carriage in the population is important to better assess the level of parasite transmission in elimination settings. The aim of this study was to detect P. falciparum gametocytes from used RDTs by quantitative PCR technique in order to use this new tool for molecular monitoring of malaria transmission. Methods: DNA was extracted from 303 RDT devices (SD Bioline Malaria Pf) using the Chelex-100 protocol. qPCR was performed in a $20 \mu \mathrm{L}$ reaction to detect and quantify transcripts of the pfs $25 \mathrm{gene}$. The cycle threshold (Ct) was determined by the emission fluorescence corresponding to the initial amount of amplified DNA. Results: We found an overall prevalence of $53.47 \%$ with an average Ct of $32.12 \pm 4.28$ cycles. In 2018, the prevalence of gametocytes was higher in the Ranérou district (76.24\%) than in the Saint-Louis district (67.33\%) where an increase in the number of gametocyte carriers in 2018 was noted, in comparison with 2017. Conclusions: RDTs are a good source of DNA for molecular monitoring of gametocyte carriage. This method, described for the first time, is a simple and effective tool to better understand the level of malaria transmission and reach elimination. Keywords: Malaria, RDT, Gametocytes, DNA extraction, Quantification, Plasmodium falciparum, qPCR.

\section{Background}

The presence of mature gametocytes (stage $\mathrm{V}$ ) in the human peripheral blood is the determining factor in the maintenance and increase of malaria transmission [1]. In Senegal, progress in the fight against malaria led the program to become part of the regional elimination of malaria by introducing primaquine in the management of the disease in the north of the country in order to reduce malaria gametocyte carriage in the population and block the transmission of the disease [2,3].

Gametocytes are sexual forms of the parasite that are transmitted from the human host to mosquito vectors and thus perpetuate the transmission of malaria $[4,5,6]$. The passage of gametocytes to vectors is possible even in sub-microscopic low-density situations in human blood (<4 gametocytes / $\mu l)[1,4,7]$. The detection and quantification of specific gametocytes from modern molecular techniques such as the quantitative Reverse Transcriptase Polymerase Chain Reaction (qRT-PCR) have shown a high frequency of sub-microscopic densities [1,7]. Even after treatment with antimalarials, the gametocytes persist for several weeks after the clearance of asexual parasite forms with longevity depending on the nature and dose of the treatment administered as well as the immune response of the host [6]. Treated subjects carrying gametocytes in their blood are potential reservoirs of the parasite [7]. The development of modern tools for the identification of these reservoirs is necessary to achieve the elimination of malaria $[4,6,8]$.

Since mosquito infection requires the presence of gametocytes in humans, knowledge of the epidemiology of gametocyte carriage is an essential parameter for assessing malaria transmission and predicting infectiousness in vectors $[1,7,8]$. Some molecular techniques are used today for the detection and quantification of gametocytes $[9,10]$. The complexity and cost of these tests limits their use in low resource areas.

Large-scale RDTs in endemic countries are now used as sources of parasite DNA [11,12] and have helped develop molecular tools to improve malaria surveillance $[13,14,15]$. Recently, numerous studies have detected asexual forms of $P$. falciparum in RDT samples by PCR [16]. However, no study has been conducted to detect and quantify gametocytes from these RDTs.

This study aimed to determine the $P$. falciparum gametocyte carriage on used RDT samples by the quantitative real time PCR (qPCR) technique. This molecular technique for detecting gametocytes from collected RDTs can be an effective tool for molecular surveillance of malaria in northern Senegal.

\section{Methods}

\section{Study area and field samples}

RDTs were collected as part of malaria surveillance in northern Senegal where the incidence of malaria is low ( $<5 \%$ o). Febrile patients who went to public or private health facilities were tested by RDTs and treated. All TDRs used (negative and positive) were stored in plastic bags at room temperature (Fig 1A).

In Senegal, malaria transmission is closely related to the rate of rainfall and generally increases during the rainy season. The density of vector populations is dependent on rainfall.

For this study, two (2) health districts of two (2) regions were concerned, including that of Saint-Louis in the Saint-Louis region and that of Ranérou in the Matam region. The large shares of malaria cases recorded in the two regions were reported in these two districts. In recent years, the district of Saint-Louis had recorded the majority of malaria cases in the Region. As part of malaria elimination, the program of Senegal began in 2018 to use primaquine combined with the usual treatment of malaria cases in this area to reduce gametocyte carriage and malaria transmission. The 2018 epidemiological data showed that the number of malaria cases in this district had doubled compared to 2017 despite the intensification of control 
strategies. In order to better appreciate the impact of primaquine on gametocyte carriage, we have chosen to study the gametocyte prevalence in 2018 in the district of Saint-Louis, which will be compared with the prevalence in 2017 before using primaquine and the prevalence in 2018 in the district of Ranérou where primaquine is not used. At the time of testing, patients were informed about the protocol and their consent was obtained.

We randomly selected a total of 303 positive RDTs from different time periods and sites. In Saint-Louis, we chose 101 positive TDRs completed in 2017 and 101 completed in 2018. In Ranérou 101 positive TDRs of 2018 were chosen.

\section{RDT devices}

The RDTs used in the northern part of the country for malaria surveillance were SD-Bioline Malaria Ag Pf for the detection of $P$. falciparum specific Histidine-Rich Protein II (HRP-II) antigen. DNA extraction was done by opening the TDR cassettes (Fig. 1C).

\section{RDT-DNA extraction methods}

Several methods of $P$. falciparum DNA extraction have been described $[11,17]$. We used the DNA extraction protocol with Chelex-100 described by Wooden J. et al (1993) which is the result of a combination of several techniques taking into account a variable number of strains of the parasite [18]. The RDTs were opened using a metal spatula to access the nitrocellulose strip. This band was taken using forceps and the blood deposition and absorption filters were cut into small pieces in labeled Eppendorf tubes (Fig. 1C). The migration zone of the blood sample on the nitrocellulose membrane was carefully scraped with a scalpel to collect the maximum amount of DNA from the devices (Fig. 1D). For each sample the material used was cleaned in $96 \%$ ethanol, then rinsed with water and dried on clean tissue paper, to minimize cross-contamination during sample preparation.

In each tube, $800 \mu \mathrm{l}$ of $0.5 \%$ Saponin solution containing $0.5 \mathrm{~g}$ of saponin and $100 \mathrm{ml}$ of PBS diluted to $1 \mathrm{x}$ were added. The tubes were then centrifuged for 10 minutes at $150 \mathrm{rpm}$ and incubated at room temperature overnight. The liquid was completely aspirated from the tubes by using a micropipette and the samples were then washed twice by adding $800 \mu$ of 1 xPBS for 5 minutes. We added $150 \mu \mathrm{L}$ of sterile water and $75 \mu \mathrm{L}$ of $20 \%$ Chelex solution into the tubes, which were tightly closed and vortexed for 15 seconds and incubated with a dry-bath incubator for 8 minutes. After centrifugation at maximum speed for 5 minutes, the supernatant containing the DNA was aspirated with a micropipette and then transferred to sterile tubes leaving the Chelex at the bottom of the tube [18].

\section{qPCR assay}

The validation of the technique was done using 6 different samples. Two (2) different RDT samples (Paracheck ${ }^{\mathrm{TM}}$-Pf and SD Bioline Malaria Ag-Pf) were made by depositing $5 \mu \mathrm{L}$ of $0.5 \%$ and $1 \%$ diluted gametocytes cultures in the RDT wells. From the $1 \%$ diluted culture $50 \mu \mathrm{L}$ were deposited on a filter paper. To these samples we added two more positive RDTs whose gametocytes with different densities were identified by microscopy and another sample of filter paper whose PCR was positive for the detection of gametocytes as a positive control. qPCR-steps were done in duplicate for samples. The SYBR Green Master Mix has been standardized to detect and quantify Pfs 25 gene transcripts using the BIO-RAD CFX96 ${ }^{\mathrm{TM}}$ tool which showed positive results.

For the Pfs25 assay we used a specific female gametocytes primers tested by Schneider et al. [19]. The Forward (5'CCATGTGGAGATTTTTCCAAATGTA, location: 253852-1253828) and Reverse (5'-catttaccgttaccacaagttaCATTC, location: 1253710-1253736) primers were used for amplification. For these primers Schneider et al. had shown that the DNA sequences of the target regions (DNA produced in vitro: ivDNA) and the cDNA were identical. The Pfs 25 primers used are specific to mature gametocytes and can be considered to quantify only female gametocytes. Schneider et al. had also shown that no significant nucleotide polymorphism were detected in the primer regions among almost 2000 samples of $P$. falciparum collected worldwide [19].

\section{Field sample analysis}

The real time-PCR was performed in a $20 \mu \mathrm{L}$ reaction. The reaction was prepared with SYBR Green Master Mix. DNA template and Nuclease-Free Water were used. The Pfs25 primers used are specific for female gametocytes and showed limited polymorphism [19]. Each $20 \mu \mathrm{L}$ reaction mixture contained $2 \mu \mathrm{L}$ of $2 \mu \mathrm{L}$ of DNA sample, $10 \mu \mathrm{L}$ of $2 x$ SYBR Green master mixtures, $2.25 \mu \mathrm{L}$ of primers at a final concentration of $300 \mathrm{nM}$ and $5.75 \mu \mathrm{L}$ of Nuclease-Free Water. Amplification included a template denaturation step at $95^{\circ} \mathrm{C}(10 \mathrm{~min})$ followed by 45 cycles of 15 seconds at $95^{\circ} \mathrm{C}$, and 1 minute at $60^{\circ} \mathrm{C}$, with fluorescence acquisition at the end of each extension step. Reactions were run in 96-well PCR plates on a Bio-Rad CFX Connect real time PCR Detection System. For our study we used 4 plates for 303 samples.

\section{Data analysis}

We used the Bio-Rad CFX Manager 3.0 software for the post-amplification data analysis.

Quantification cycle $(\mathrm{Cq})$ or cycle threshold (Ct) determination mode was set to single threshold with baseline-subtracted curve fit and a user defined threshold of 50 relative fluorescence units (RFUs) for analysis of parasite. A cycle threshold value (Ct) $<35$ was chosen for positive PCRs. Samples with a cycle value greater than $35 \mathrm{Ct}$ were considered negative for PCR. The data was also exported to Excel and analyzed with the Epi Info software. 


\section{Results}

\section{Amplification of pfs 25 gene by quantitative real-time PCR}

We standardized a qualitative and conventional qPCR protocol to detect pfs 25 gene transcripts in RDT samples. With this method, 162 of the 303 RDT samples analyzed (53.47\%) were positive (Table 1). Negative samples did not show gene amplification or showed amplification after 35 cycles $(n=141)$. (Fig 2A)

The $\mathrm{Ct}$ is the threshold point at which the fluorescence signal is significantly greater than the background noise, i.e., the minimum number of cycles for which the amplified DNA is detectable. The average Ct was 32.12 with standard deviation of 4.28 . The minimum number of cycles observed for DNA amplification was 8 cycles corresponding to the presence of a very large initial amount of parasite DNA (Fig. 2D).

\section{Gametocyte carriage}

Amplification of the Pfs 25 gene allowed the detection of gametocytes on each RDT. The fluorescence emission determines a Ct value that reflects the initial amount of DNA (2B). When the $\mathrm{Ct}$ is low the initial amount of DNA is large. For the qPCR positive samples ( $\mathrm{n}=162)$ gene amplification was shown before 35 cycles. The overall gametocyte prevalence was 53.47\% (162/303) (table 1$)$.

Table 1 : Gametocyte carriage by study area and by year of samples collection.

\begin{tabular}{|c|c|c|c|c|c|}
\hline \multirow[t]{2}{*}{ RESULTS } & \multicolumn{3}{|c|}{ SITES } & \multirow[t]{2}{*}{ TOTAL } & \\
\hline & Ranérou 2018 & Saint-Louis 2017 & Saint-Louis 2018 & & \\
\hline Negative & 24 & 84 & 33 & 141 & 2018 the gametocyte prevalence was $76.24 \%$ \\
\hline$\%$ & $23,76 \%$ & $83,17 \%$ & $32,67 \%$ & $46,53 \%$ & $(77 / 101)$ in the Ranérou district and $67.33 \%(68 / 101)$ in the \\
\hline Positive & 77 & 17 & 68 & 162 & ouis district (Table 1 ). The results of the analysis \\
\hline$\%$ & $76,24 \%$ & $16,83 \%$ & $67,33 \%$ & $53,47 \%$ & lence in the \\
\hline TOTAL & 101 & 101 & 101 & 303 & Louis was lower with $16.83 \%$ (17/101). A large \\
\hline
\end{tabular}

year primaquine was combined with antimalarial treatment to reduce the gametocyte carriage. Increased gametocyte carriage despite the use of primaquine makes it necessary to improve the strategy against malaria transmission.

\section{Discussion}

We have performed a DNA extraction technique from RDT samples to detect expression of $P$. falciparum Pfs 25 gene by qPCR. This study confirms the thesis that RDTs are sources of that $P$. falciparum mature gametocytes DNA. In addition to quantifying the number of DNA amplification cycles, SYBR Green makes it possible to estimate the number of gametocyte copies $[9,20,21]$. The number of copies would have allowed providing more precision on the estimation of the gametocyte density in this study [21]. The large-scale implementation of molecular techniques for the detection of the parasite's sexual forms is an effective means to control epidemiology of gametocyte carriage and to get an estimate of malaria transmission [9,22]. The DNA extraction method from RDTs is simple and can be applied on the field for molecular control of transmission with a rapid estimate of the level of gametocytes carriage.

Overall, qPCR analyzed 94 RDT samples per plate and is of high epidemiological significance. The method is reproducible and easily adaptable to determine the carriage of gametocytes from large-scale RDTs used for the diagnosis of malaria. The detection of mature gametocytes of $P$. falciparum by qPCR was carried out only from DNA extracted from filter papers or directly from blood. The qPCR is a sensitive technique which can detect sub-microscopic densities of mature gametocytes on both filter paper and RDTs [9]. This study shows an overall gametocyte prevalence of $53.47 \%(162 / 303)$ with a mean Ct of 32.12 cycles with a standard deviation of 4.28 . This result expresses the level of parasite transmission from humans to mosquitoes and allows an assessment of the risk of transmission of the disease. In the St. Louis district, gametocyte prevalence was higher in 2018 than in 2017. In recent years, larger population movements in search of work from high-transmission areas (south of the country) to low-transmission areas (in the north) has contributed to an the increased transmission of malaria in this northern part of the country [3].

However, this increase in the level of transmission can also be explained by the duration of strike action on the part of health staff which led to an interruption in the operational implementation of control strategies and an increase in malaria cases. The artemisinin-based combination therapy (ACTs) used in the treatment of malaria cases promote the production of gametocytes. More malaria cases treated entail more gametocyte carriers. The Saint-Louis region has experienced excessive rainfall in 2018 as compared to 2017 when a deficit in rainfall was recorded depressed [23,24]. This increase in rainfall in 2018 led to an increase in the Anopheles population and the level of malaria transmission.

The increase of gametocyte carriers is a real problem for the parasite reservoir control as an element of the malaria prevention strategy. The collected RDTs analysis in the Matam area (Ranérou district) showed a gametocyte prevalence of $67.33 \%$ (68/101). According to the National Malaria Control Program, this district has for many years recorded the vast majority of reported cases in the region where the transmission of the disease is strong at 
the local level [3]. Future studies involving asymptomatic patients are needed to evaluate the sensitivity and accuracy of the method and to evaluate the potential benefits of molecular control of transmission [8]. Extension of this study to the 3 regions where the malaria elimination project is operational will provide reliable estimates to better guide malaria control strategies [1]. The use of RDTs as a source of DNA for the detection of mature gametocytes usin molecular techniques is relevant for epidemiological studies assessing the efficacy of candidate vaccines against malaria transmission $[8,22]$ and it is, therefore, an important support for the control of malaria reintroduction $[8,9]$.

\section{Conclusions}

The collection of RDTs in the field is simple and systematic in Senegal for the surveillance of malaria. The use of this tool to determine the prevalence of gametocytes is practical and affordable. The development of this method with a large-scale estimation of gametocyte density, including on asymptomatic individuals, will increase the monitoring power and allow further elimination. PCR is a tool with good sensitivity for detection of gametocyte genes expression [21]. The pressure of the drugs on the parasite contributes to the production of gametocytes and is responsible for the presence of these parasitic sexual forms in the blood of treated subjects.. Development of effective tools to monitor the carriage of gametocytes is necessary to control parasite pools. The recent use of fluorescence for the detection of gametocyte DNA has led to a better description of the male and female gametocyte genes [25]. We researched the amplification of the Pfs 25 gene to determine the prevalence of gametocytes in northern Senegal. Our conclusion is that this method should be put to greater use to determine the level of gametocyte carriage in the larger population by including asymptomatic individuals in order to develop adequate strategies to destroy the parasite's reservoirs and achieve malaria elimination [16] Primaquine has been administered only to malaria cases. A mass administration of primaquine will have an impact on gametocyte carriage and will help curb malaria transmission.

\section{Abbreviations}

RDTs. Rapid diagnostic tests for malaria - cDNA: Complementary DNA - qPCR. quantitative real time PCR - Cq. Quantification cycle - Ct: Cycle threshold - RFUs: Relative Fluorescence - ACTs: Artemisinin-based Combination Therapy

\section{Declarations}

\section{- Ethics approval and consent to participate}

The study protocol was submitted for approval to Senegal's National Committee for Ethics for Health Research (CNERS) (Reference: 046/2015/CER/UCAD).

\section{- Consent for publication}

"Not applicable"

\section{- Availability of data and materials}

The datasets used during the current study are available from the corresponding author on reasonable request.

\section{Competing interests}

The authors declare that they have no conflict interests.

\section{Funding}

This work was supported by MACEPA for the field survey and sample collection. The Department of Medical Parasitology at Cheikh Anta Diop University supported the analysis of the samples and the interpretation of the results.

\section{- $\quad$ Authors' contributions}

KT Guiguemde, A Lam, MP Diouf, IA Manga, M Ndiaye and A Collé Lô developed the DNA extraction protocol and performed the manipulations of the samples in the laboratory. RCK Tine and B Faye supervised the work and validated the results. G Dieng Sow, M Diop, T Souane are the focal points of MACEPA in the northern regions who led the field investigations under the coordination of $Y$ Dieye and taking part in interpretation and analysis of data as well as writing. All authors read and approved the final manuscript.

\section{Acknowledgements}

The authors acknowledge the team of the Parasitology laboratory of Cheikh Anta Diop University for their collaboration.

\section{Authors' information}


Professor B Faye is the Head of Department of Medical Parasitology at Cheikh Anta Diop University in Dakar where Professor RCK Tine, KT Guiguemde, A Lam, MP Diouf, IA Manga, M Ndiaye and A Collé Lô work. Y Dieye is the coordinator of the Malaria Control and Evaluation Partnership (MACEPA) in Senegal. G Dieng Sow, M Diop and T Souane are the focal points of MACEPA in the northern regions of Senegal.

\section{References}

1 Coalson JE, Walldorf JA, Cohee LM, Ismail MD, Mathanga D, Cordy RJ, Marti M, et al. High prevalence of Plasmodium falciparum gametocyte infections in school-age children using molecular detection: patterns and predictors of risk from a cross-sectional study in southern Malawi. Malar $J$. 2016; 15 (1): 527. doi:10.1186/s12936-016-1587-9. http://dx.doi.org/10.1186/s12936-016-1587-9.

2 Greenwood BM. Control to elimination: implications for malaria research. Trends in Parasitology. 1 oct 2008;24(10):449-54. doi: https://doi.org/10.1016/j.pt.2008.07.002

3 PATH, Malaria Control and Elimination Partnership in Africa. Sénégal : Vers l'élimination du paludisme. PATH. 2018.

4 Santolamazz F, Avellino P, Siciliano G, Yao FA, Lombardo F, Ouédraogo JB, Mangano VD. Detection of Plasmodium falciparum male and female gametocytes and determination of parasite sex ratio in human endemic populations by novel, cheap and robust RTqPCR assays. Malar J. 2017; 16(1), 468. doi: 10.1186/s12936-017-2118-z

5 Kefi M, Mavridis K, Simões ML, Dimopoulos G, Siden-Kiamos I, Vontas J. New rapid one-step PCR diagnostic assay for Plasmodium falciparum infective mosquitoes. Scientific Reports. 2018; 8(1):1462.

6 WWARN Gametocyte Study Group. Gametocyte carriage in uncomplicated Plasmodium falciparum malaria following treatment with artemisinin combination therapy: a systematic review and metaanalysis of individual patient data. MC Medicine. 2016, 14:79. doi: 10.1186/s12916-016-0621-7.

7 Schneider P, Bousema JT, Gouagna LC, Otieno S, van de Vegte-Bolmer M, Omar SA, et al. Submicroscopic Plasmodium falciparum gametocyte densities frequently result in mosquito infection. Am J Trop Med Hyg. 2007; 76:470-474.

8 Taylor BJ, Lanke K, Banman SL, Morlais I, Morin MJ, Bousema T, Rijpma SR, Yanow SK. A Direct from Blood Reverse Transcriptase Polymerase Chain Reaction Assay for Monitoring Falciparum Malaria Parasite Transmission in Elimination Settings. Am J Trop Med Hyg. 2017; 97(2):533-543. doi: 10.4269/ajtmh.17-0039

9 Pett H, Gonçalves BP, Dicko A, Nébié I, Tiono AB, Lanke K, and al. Comparison of molecular quantification of Plasmodium falciparum gametocytes by Pfs25 qRT-PCR and QT-NASBA in relation to mosquito infectivity. Malar J. 2016 15:539. Doi : 10.1186/s12936-016-1584-z

10 Coalson JE., Walldorf JA, Cohee LM, Ismail MD, Mathanga D, Cordy RJ, Marti M, et al. High prevalence of Plasmodium falciparum gametocyte infections in school-age children using molecular detection: patterns and predictors of risk from a cross-sectional study in southern Malawi. Malar $J$. 2016 ; 15 (1): 527. doi:10.1186/s12936-016-1587-9. http://dx.doi.org/10.1186/s12936-016-1587-9.

11 Morris U, Aydin-Schmidt B, Shakely D, Mårtensson A, Jörnhagen L, Ali AS, et al. Rapid diagnostic tests for molecular surveillance of Plasmodium falciparum malaria -assessment of DNA extraction methods and field applicability. Malar J. 2013;12(1):106. doi : https://doi.org/10.1186/1475-287512-106

12 Veron V, Carme B: Recovery and use of Plasmodium DNA from malaria rapid diagnostic tests. AmJ Trop Med Hyg. 2006; 74:941-943.

13 Cnops L, Boderie M, Gillet P, Van Esbroeck M, Jacobs J: Rapid diagnostic tests as a source of DNA for Plasmodium species-specific real-time PCR. Malar J. 2011, 10:67.

14 Ishengoma DS, Lwitiho S, Madebe RA, Nyagonde N, Persson O, Vestergaard LS, Bygbjerg IC, Lemnge MM, Alifrangis M: Using rapid diagnostic tests as source of malaria parasite DNA for molecular analyses in the aera of declining malaria prevalence. Malar J. 2011, 10:6.

15 Robinson A, Busula AO, Muwanguzi JK, Powers SJ, Masiga DK, Bousema T, et al. Molecular quantification of Plasmodium parasite density from the blood retained in used RDTs. Scientific Reports. 2019; 9(1):5107. https://doi.org/10.1038/s41598-019-41438-0

16 Wargo AR, Randle N, Chan BH, Thompson J, Read AF, Babiker HA. Plasmodium chabaudi: reverse transcription PCR for the detection and quantification of transmission stage malaria parasites. Exp Parasitol. 2006;112(1):13-20. doi: 10.1016/j.exppara.2005.08.013

17 Ishengoma DS, Lwitiho S, Madebe RA, Nyagonde N, Persson O, Vestergaard LS, Bygbjerg IC, Lemnge MM, Alifrangis M. Using rapid diagnostic tests as source of malaria parasite DNA for molecular analyses in the era of declining malaria prevalence. Malar J. 2011;10:6. doi: 10.1186/14752875-10-6

18 Wooden J, Kyes S, Sibley CH. PCR and strain identification in Plasmodium falciparum. Parasitology Today. 1993;9(8):303-5. https://doi.org/10.1016/0169-4758(93)90131-X 
19 Schneider P, Reece SE, Van Schaijk BCL, Bousema T, Lanke KHW, Meaden CSJ, et al. Quantification of female and male Plasmodium falciparum gametocytes by reverse transcriptase quantitative PCR. Mol Biochem Parasitol. 2015; 199:29-3.

20 Colborn JM, Byrd BD, Koita OA, and Krogstad DJ. Estimation of Copy Number using SYBR Green: Confounding by AT-rich DNA and by Variation in Amplicon Length. Am. J. Trop. Med. Hyg. $2008 ; 79(6): 887-892$.

21 Lima NF, Bastos MS and Ferreira MU. Plasmodium vivax: reverse transcriptase real-time PCR for gametocyte detection and quantitation in clinical samples. Exp Parasitol. 2012 ; 132(3): 348-354. doi:10.1016/j.exppara.2012.08.010.

22 Contamin H, Fandeur T, Bonnefoy S, Skouri F, Ntoumi F and Mercereau-Puijalon O. PCR Typing of Field Isolates of Plasmodium falciparum. J. Clin. Microbiol. 1995; 33(4): 944-951.

23 Direction de l'Analyse, la Prévision et les Statistiques agricoles (DAPSA). Rapport d'évaluation préliminaire des récoltes 2018/2019. 2018. http://www.dapsa.gouv.sn/sites/default/files/Rapport\%20situation\%20campagne\%202018\%20et\%20r\%C3\%A9sultats\%20pr\%C3\%A9liminaires_1.pdf Accessed 28 Sept 2019

$24 \mathrm{Ba}$ DD, Ndiaye PM, Faye C. Variabilité pluviométrique et évolution de la sécheresse climatique dans la vallée du fleuve Sénégal. Revue Togolaise des Sciences. $2018 ; 12(1)$.

25 Meerstein-Kessel L, Van der Lee R, Stone W, Lanke K, Baker DA, Alano P et al. Probabilistic data integration identifies reliable gametocyte-specific proteins and transcripts in malaria parasites. Sci Rep. 2018; 8(1):410. doi: 10.1038/s41598-017-18840-7. doi: 10.1038/s41598-017-18840-7.

\section{Figures}

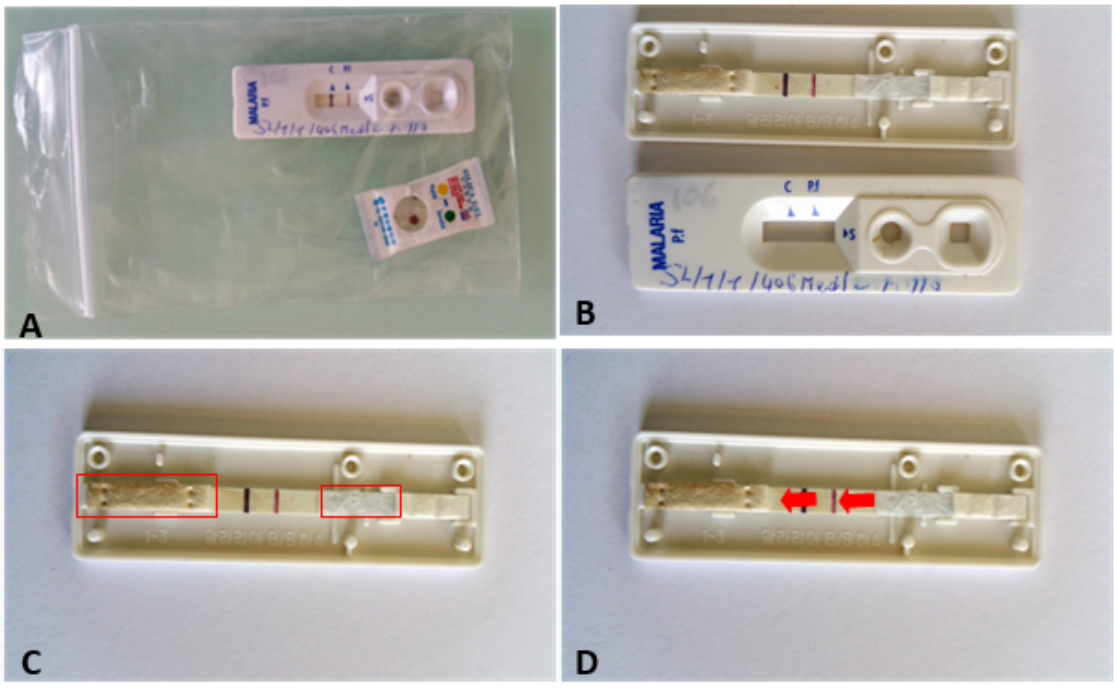

\section{Figure 1}

RDT device and fragments used for DNA extraction. (A) SD-Bioline RDT device stored in plastic bag; (B) device opened, (C) absorption filters on nitrocellulose strip, (D) blood migration zone scraped. 

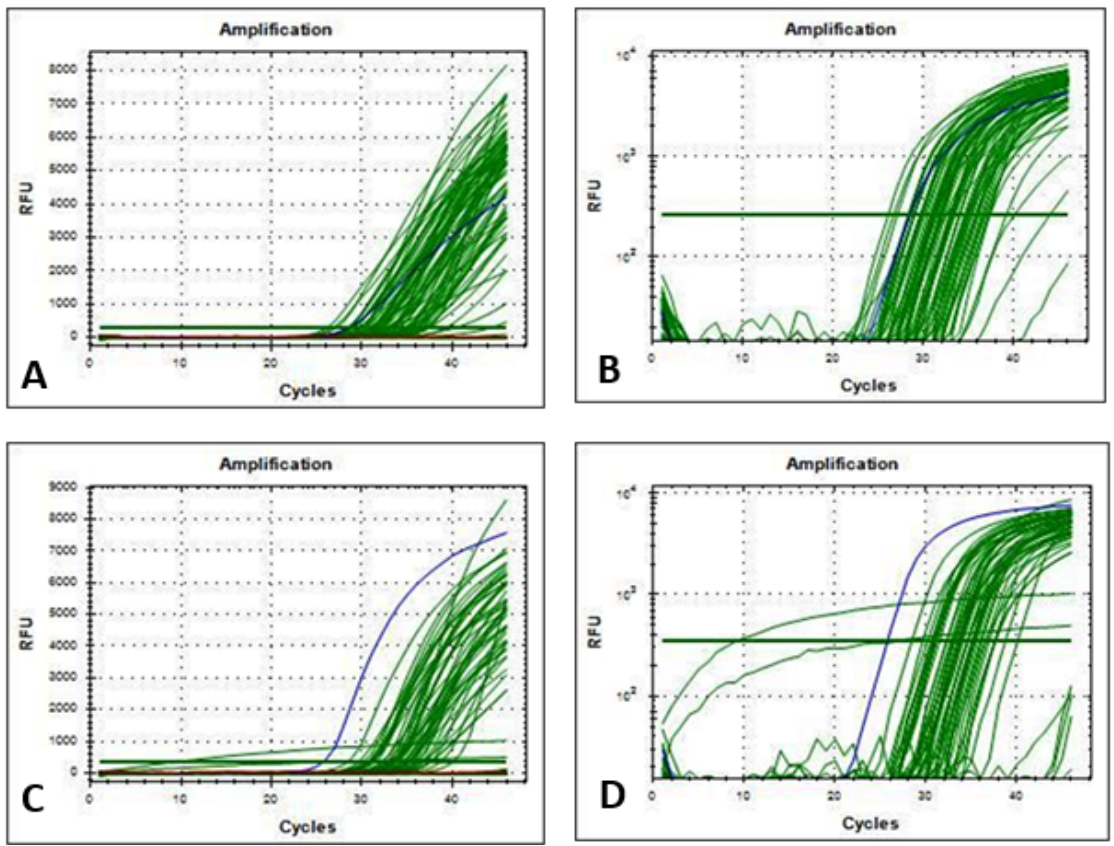

\section{Figure 2}

Plasmodium falciparum Pfs25 detection from RDT by direct real time-PCR. (A) DNA amplification kinetics per cycle on plate 1 for each sample (B) logarithmic value of fluorescence and presence of background noise (C) Amplification on plate 2, (D) DNA amplification at 8 cycles. Reactions performed on a conventional qPCR instrument (Bio-Rad CFX Connect) with a threshold setting of 50 relative fluorescence units (horizontal line). 